\title{
A behavioural model for the level crossing collision risk assessment
}

\author{
M. Ghazel \\ INRETS/ESTAS-The French National Institute of Transport and Safety \\ Research, France
}

\begin{abstract}
Level crossings (LC) are identified as being critical security points in both road and rail infrastructures. Statistics show that more than three hundred people are killed every year in Europe in more than 1200 accidents occurring at level crossings.

In this paper, we propose and apply a systematic method to establish a global model involving both rail and road traffic in the level crossing area. The model building approach proposes a progressive integration of elementary models, each of which describing the behaviour of a part in the whole LC environment. We are interested more precisely in a particular phenomenon which may cause collisions at level crossings, and which corresponds to the accumulation of vehicle waiting queues on the LC exit zone. As a model, we use Petri Nets (PN). For the sake of precision and in order to obtain a realistic and fine behavioural model, quantitative aspects are integrated in the form of functions (stochastic distributions, deterministic values, probabilities, etc.) associated to the transitions of our PN model $(\Rightarrow$ Stochastic PN).
\end{abstract}

Keywords: level crossing, risk assessment, modelling, Petri Net.

\section{Introduction}

A level crossing (LC) is an intersection point (at the same level) between railway and road traffic. In areas with high traffic density, level crossings are generally fitted with automatic protection systems (lights, barriers, alarms).

In terms of safety, LCs have been identified as being weak points in the railway and road transport networks. Every year more than 300 people are killed in more than 1200 accidents occurring at LCs through Europe. We have to recall that in France, as well as in most of the other European countries, under nominal condi- 
tions railway traffic has absolute priority when a train is crossing through a LC, i.e trains don't have to stop or to be sure that the way is free before crossing a LC.

A problem that has caused several train/vehicle collisions at LC is when a waiting queue is formed in the exit area of the LC. Indeed, it has been shown that in general when the LC is open (barriers in the high position and green road lights showing), and when a traffic jam occurs on the exit of the LC, road users arriving to the LC do not stop before the protection barrier, but enter to the LC crossing zone $(\mathrm{CZ})$, thus risking to remain blocked on the rail track until a train arrives. This is obviously forbidden in the highway code, as even if the LC is open to the road traffic, road users have to insure that they can leave the $\mathrm{CZ}$ safely before going in.

Let us note that there is a system based on inductive loops enabling to detect traffic jams. However, the deployment of this system is not generalized and above all, in general this kind of system becomes out of order with roadworks, which are a main cause of jams.

In this paper, we propose to develop a complete model integrating both the railway traffic and the road traffic. We emphasize more particularly the phenomenon of traffic jam in the LC exit zone. Our goal is to prepare a basis (behavioural model) for the evaluation of the collision risk on LCs induced by these circumstances.

The behavioural model we develop is a Petri Net (PN) model and the approach we implement is progressive. First, we work out elementary models each describing a part of the LC environment. These models are then assembled, thus obtaining a global model. The description of our behavioural model is then refined in order to obtain a precise and realistic description.

The paper is organized as follows: in section 2, a description of the general LC topography and its environment is proposed. We develop in section 3 a model describing the $\mathrm{LC}$ environment starting from elementary models of the numerous parts of the system. Finally, in section 4, we conclude the study by reviewing the main steps of the modelling approach proposed and we give some guidance for future work.

\section{LC environment topology}

LC environment designates all the parts involved in the dynamics of the rail/road junction. A LC can be guarded or not. Generally, only LCs with low traffic moment (road traffic frequency * rail traffic frequency) are not protected. The others are generally equipped with automatic protection systems which may comprise train sensors, road lights, sound alarms and barriers. In our study we consider a standard automatic LC in the intersection of a single railway track with a bidirectional road, equipped with train sensors, road lights, sound alarms and two half-barriers (cf. figure 1).

The LC protection is performed in such a way that when a train approaching the $\mathrm{LC}$ is detected by the train sensor, the closure cycle is initiated. The LC is reopened to the road traffic as soon as the train is detected in the departure direction. 


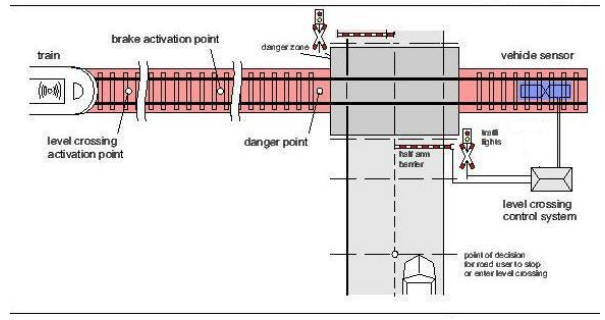

Figure 1: General topography of an automatic LC.

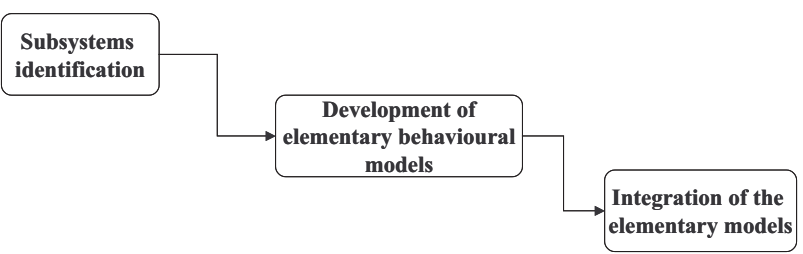

Figure 2: Steps of the modelling approach.

\section{Behavioural model}

In order to obtain a complete behavioural model of the LC environment, a progressive approach will be employed [5]. First, the studied environment will be decomposed in subsystems (modules). Then, functional requirements of each of these modules are expressed in a semi formal way and elementary behavioural models are set up for all the subsystems. The global model is finally obtained by integrating the elementary models while establishing the behavioural interdependencies. This approach (cf. figure 2) was developed in [4].

By analyzing the dynamics of the studied system, three main parts can be identified: road traffic, railway traffic and LC control. In section 3.1, we will develop elementary models of the identified subsystems. Then in section 3.2, interactions between the subsystems behaviours will be taken into account in such a way that the elementary models are integrated in a same global one. The integration step is done by employing mechanisms similar to those developed in [4].

The formalism used during the modelling step is that of Petri Net (PN) for the advantages it offers in terms of semantics and expression ability. Also, basing our study on models suitable for formal analysis and simulation is important in the framework of safety studies [6], [7]. More precisely, the PN category which will be used in our study is that of stochastic Petri Nets (SPN) [1] in such a way that the dynamics can be described precisely by associating time firing functions (probabilistic functions, deterministic values, etc.) and probability values to transitions [2]. Moreover, using inhibitor arcs has the advantage of avoiding a useless complexity on our model. An inhibitor arc links a place $P$ to a transition $T$. By default, 
as for usual arcs, an inhibitor arc has as weight 1 . It prevent the transition $T$ firing as long as $P$ is marked. It can also have a weight $n$, in this case it forbids the firing of this transition while there is at least $n$ tokens in the place. Finally, note that in this section only the global structure of the behavioural model is explained. Refining of this model is made while introducing temporal and probabilistic dynamic aspects. For lack of space this part is not discussed in this paper.

\subsection{Elementary models}

\subsubsection{Modelling of the railway side}

In this section, we will depict the railway traffic under the hypothesis that we are dealing with a single track line. Our model can be easily transposed, modulo some adaptations, to describe a double track traffic. Moreover two kinds of trains are considered: passenger trains and freight trains; according to the train type, the speed is different. Finally we suppose that there is no overlap between consecutive train traversals.

The elementary model set for railway traffic is presented in figure 3-left. In this model $t_{1}$ allows the generation of trains arriving towards the LC, $t_{2}$ corresponds to passenger trains selection (with a given probability $p_{\text {passenger }}$ ) and $t_{3}$ to the selection of freight trains (with a probability $p_{\text {freight }}=1-p_{\text {passenger }}$ ). $t_{4}$ corresponds to the arrival of a passenger train to the LC and $t_{5}$ that of a freight train. Finally $t_{6}$ accounts for train detection at the LC exit.

\subsubsection{Modelling of the road side}

In order to determine the risk exclusively related to the traffic jam problem, our model (cf. figure 3-right) does not take into account other road users' behaviours which can also cause accidents, like for instance when a vehicle goes in zigzag

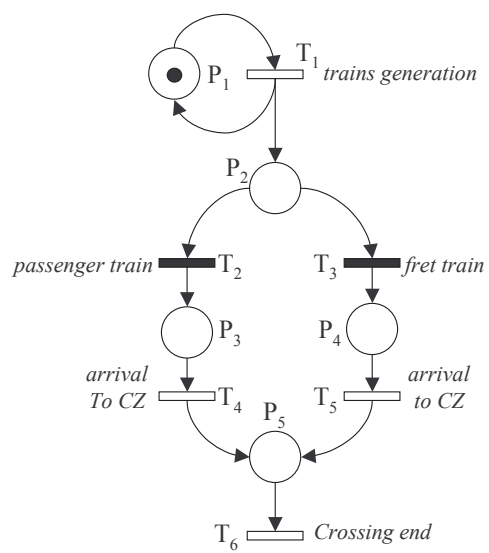

Figure 3: Elementary models of the rail (left) and road (right) traffics. 
through a closed LC. Moreover, only one direction of the road traffic is modelled here, since in our study we consider that the jam risk exists on only one running direction.

In the model of figure 3-right, $T_{8}$ enables the generation of vehicles at the LC entry. The left branch following place $P_{7}$ describes the situation where there is no jam on EZ. At most two vehicles can be on the CZ simultaneously. This corresponds to the place-invariants respectively on $\left\{P_{8}, P_{9}\right\}$ and $\left\{P_{10}, P_{11}\right\}, M\left(P_{8}\right)+$ $M\left(P_{9}\right)=M\left(P_{10}\right)+M\left(P_{11}\right)=1$. As two vehicles can follow each other when they cross $\mathrm{CZ}$, the traversal is depicted with 2 places $\left(P_{8}\right.$ et $\left.P_{10}\right)$ and 3 transitions ( $T_{9}, T_{10}$ et $T_{11}$ ). Transition $T_{9}$ stands for the traversal start (access to CZ), $T_{10}$ corresponds to the $\mathrm{CZ}$ exit start and $T_{11}$ to the complete exit of $\mathrm{CZ}$ and the entrance to EZ. EZ is the exit zone of the LC at which some traffic jams can arise. The part of the model following $T_{11}$ represents the vehicle places in EZ (each position being described with a place $P_{12}^{i}, i: 1 \longrightarrow N$ ). Place $P_{13}$ models EZ capacity and $P_{20}$ stands for a counter of which marking indicates the number of vehicles in EZ $\left(M\left(P_{20}\right)=\sum_{i} M\left(P_{12}^{i}\right)\right)$. Despite the information given by $P_{20}$ marking is redundant with that of places $P_{12}^{i}, P_{20}$ in combination with some inhibitor arcs are implemented in order to simplify the model and to make it clearer. Concretely, the $N$-inhibitor arc linking $P_{20}$ to $T_{9}$ prevents the firing of $T_{9}$ when EZ is saturated (i.e when the maximum capacity of this zone is reached). Hence, firing $T_{9}$ denotes the fact that the crossing vehicle does not risk to remain blocked on CZ. If we denote $N$ the EZ capacity, we can obviously discern 2 place-invariants: $M\left(P_{13}\right)+M\left(P_{20}\right)=N$ and $M\left(P_{13}\right)+\sum_{i} M\left(P_{12}^{i}\right)=N$.

As to the right branch after $P_{7}$, it describes the scenario when a jam occurs on EZ i.e, when the maximum capacity $N$ of this area is reached (there are simultaneously $N$ vehicles in EZ). Thanks to the inhibitor arc linking $P_{13}$ to $T_{13}, T_{13}$ which is an immediate transition, cannot fire if there is still at least one token in $P_{13}$, i.e EZ is not saturated. Indeed as $\operatorname{sSum}_{i} M\left(P_{12}^{i}\right)+M\left(P_{13}\right)=N, M\left(P_{13}\right)=0 \Rightarrow$ $\sum_{i} M\left(P_{12}^{i}\right)=N$, i.e EZ is saturated. In this case, two progresses can be envisaged: the first when the vehicle adopts a risky behaviour and enters to $\mathrm{CZ}$ despite of EZ saturation, that is represented by $T_{14}$ to which a custom probability value $p_{\text {risk }}$ is assigned. This behaviour is unaffectedly forbidden in the highway code. The second evolution corresponds to the situation where the vehicle awaits at the LC entry until a position becomes free in EZ. That corresponds to the firing of $T_{15}$ and the wait in $P_{15}$. A custom probability $p_{\text {safe }}=1-p_{\text {risk }}$ is associated to $T_{15}$. The $N$-weighted inhibitor arc $P_{20} \rightarrow T_{16}$ prevents $T_{16}$ firing as long as there are $N$ tokens in $P_{20}$. In other words, the waiting vehicle does not proceed as long as EZ is saturated. $P_{24}$ insures that no more than one vehicle can be in the $\mathrm{CZ}$ entrance and finally $T_{22}, P_{25}$ and their associated arcs enable to reserve the first place in $\mathrm{CZ}$ (token in $P_{9}$ ) to the vehicle waiting in $P_{15}$.

\subsubsection{Modelling of the control part}

The operation of the LC automatic protection system is the following: when a warning is launched, that is a train is detected in the arrival direction, the orange traffic lights start flashing for 4 seconds and the sound alert start ringing, then 


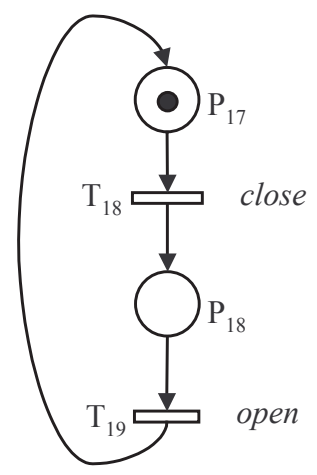

Figure 4: Elementary model of the LC control system.

simultaneously the red traffic lights start showing and the barriers move down. The red lights remain switched on and the barriers are kept in the low position until the train is detected (by train sensors) in the leaving direction. Thus the red lights are switched off, the sound alert is stopped and the half-barriers are raised.

LC protection system operation will be roughly described in order to point out closure and opening states. The LC is considered closed to the road traffic as soon as the red lights start showing and is again open to the road traffic when the barriers are completely raised after the train has left the LC (cf. figure 4).

Initially, the LC is open to the road traffic ( $P_{17}$ marked). $T_{18}$ corresponds to the barriers downgrade, $P_{18}$ to the closed state and $T_{19}$ to the LC opening. The operation of the other subsystems (road lights, sound alarm) is not shown in our model. Furthermore the protection system is supposed to operate normally.

\subsection{Global model establishment}

Integrating the elementary models in order to obtain a global behavioural model is made by introducing the interactions between subsystems behaviours. In [4] we propose a progressive method which enables representing these dependencies in a progressive way in the shape of constraints linking the behavioural elementary models. We will take advantage of this method in our study.

The first interaction is between the railway traffic and the LC control system: As soon as a train is detected in the arrival direction (with train sensors linked to the local control system), the closure cycle is launched. The LC is considered to be closed to the road traffic as soon as the red lights show, that is $4 \mathrm{~s}$ after the alert is given. This is performed with a new place $P_{a}$ between $T_{1}$ and $T_{18}$ and the temporal constraint $[4,4]$ as firing interval associated to $T_{18}$. Conversely, as soon as the train leaves $\mathrm{CZ}$, the $\mathrm{LC}$ is re-opened to the road flow, which is represented by introducing place $P_{d}$ between $T_{6}$ and $T_{19}$ as well as the firing interval $[6,6]$ associated to $T_{19}, 6 s$ being the time duration necessary for the barriers to be raised. 


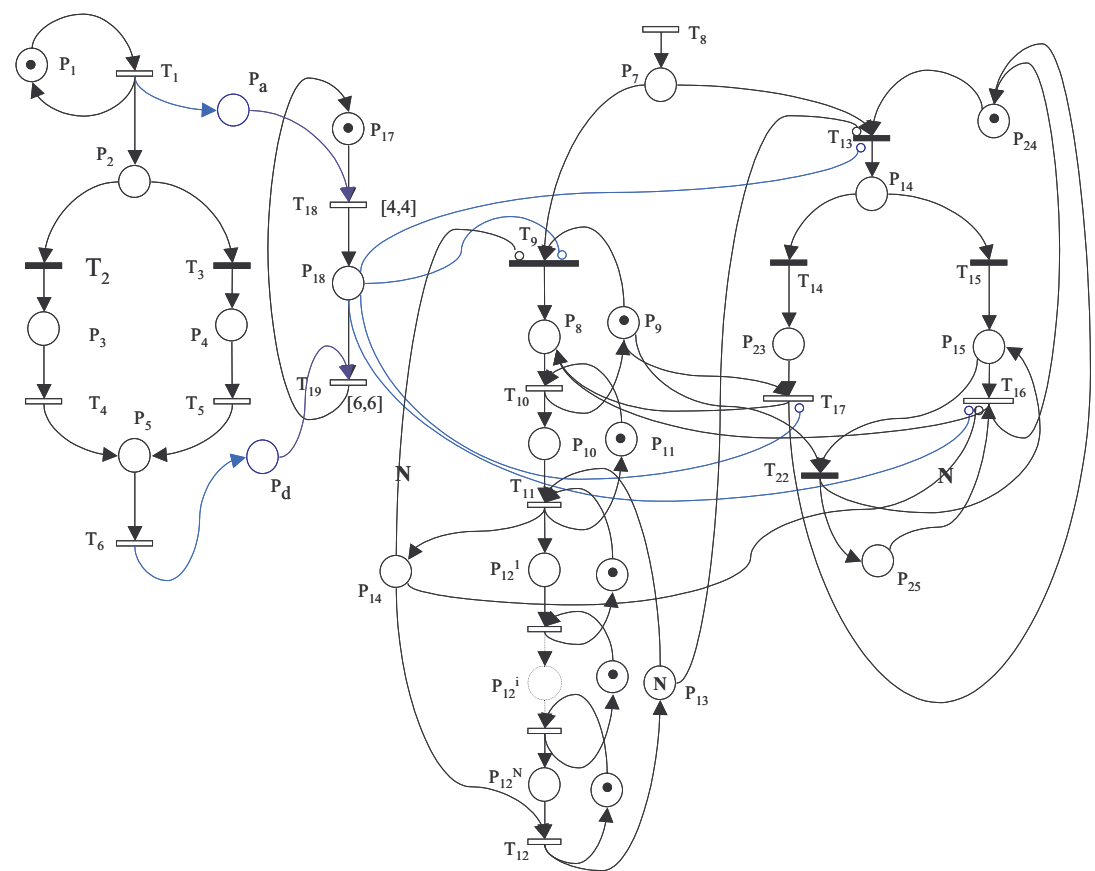

Figure 5: The global behavioural model.

The second interaction is between the local control system and the road traffic. Indeed, the control system is responsible of managing the road traffic across the LC by either allowing or blocking the traffic. This interdependence is translated by preventing the firing of transitions which correspond to the access into $\mathrm{CZ}$ as long as the LC is closed to the road traffic. In our model, this is performed with inhibitor arcs linking $P_{18}$ respectively to $T_{9}, T_{13}, T_{16}$ and $T_{17}$. Finally, let us note that there is no direct interaction between the railway traffic and the road traffic as the LC control system is responsible of managing the circulation through the LC and acts as an interface between the railway and road traffics.

The global behavioural model obtained is thus shown on figure 5 below.

For lack of space, we do not deal with dynamics simulation. We restrict the scope of this paper to discuss the behavioural model establishment. This model is then enriched (stochastic distributions, customer probabilities) in order to reflect in a realistic way the system behaviour. The goal behind the modelling phase is to assess quantitatively the collision risk. The influence of several parameters on the collision risk will be studied. These parameters are:

- the length $N$ of the vehicles queue on EZ area, in other terms the distance between the LC and the origin of the traffic jam. In our model, this will be performed by varying $N$, the number of places $P_{12}^{i}$; 
- the $p_{\text {risk }}$ rate which reflects the proportion of vehicles entering the $\mathrm{CZ}$ despite a jam being formed on EZ;

- the value $\mathcal{D}$ of the opening duration of the traffic in each direction.

Let us note that among the parameters listed above, only the last one is rulable and can be fixed by the roadworks manager. Simulation will thus allow us to estimate in a quantitative manner the impact of the opening/closing duration on the collision risk level.

\section{Conclusion}

In this paper a risk assessment model dealing with collisions on LC is proposed. The development of the behavioural model was made progressively by integrating the elementary models describing the behaviours of the various parts in the LC environment. Modelling was quite complex as various phenomena have to be depicted and given the interactions between the actors' behaviours within the LC environment.

The modelling approach proposed is distinguished by its gradual implementation. From an application point of view, the elaborated models take into account the behaviours of the LC protection system, of the road traffic and of the railway traffic, together. Moreover, the quantitative specification of behaviours offers a precise description and consequently realistic results. We showed the interest of having a formal model in order to set up some criteria affecting the safety, in an optimal way.

Among the perspectives of this work, it will be interesting to automatize some tasks in the modelling process. The idea is to work up generic patterns [3] which, by instantiation, enable to describe certain behavioural aspects and requirements. The elaboration of a part or the whole of the behavioural model can thus be obtained by assembling these instances. In [4], we propose some outlines concerning particularly the interdependencies between elementary behaviours. We wish to investigate additional methods in order to make some modelling tasks systematic. Finally, we want to develop evaluation techniques for stochastic PN models for which there are no analytical methods to make evaluation more efficient.

\section{References}

[1] M. Ajmone Marsan Stochastic Petri nets: An elementary introduction Advances in Petri Nets 1989, Lecture Notes in Computer Science, G. Rozenberg, Ed. Springer Verlag, 1990, vol. 424, pp. 129.

[2] M. Ajmone Marsan and G. Chiola On Petri nets with deterministic and exponentially distributed firing times Advances in Petri Nets 1987, Lecture Notes in Computer Science, G. Rozenberg, Ed. Springer Verlag, 1987, vol. 266, pp. $132-145$.

[3] E. Gamma, R. Helm, R. Johnson,J. Vlissides Design Patterns, Elements of Reusable Object-Oriented Software, Addison-Wesley, 1995. 
[4] M. Ghazel and E.M El-Koursi Automatic Level Crossings: From Informal Functional Requirements' Specifications to the Control Model Design, Proceedings of IEEE-SoSE2007, Texas-USA, 16th-18th April 2007.

[5] M. Ghazel, A. Toguyéni and M. Bigand An UML approach for the metamodelling of automated production systems for monitoring purpose, Computers in Industry (CiI), vol. 55, pp. 283-299, Ed. Elsevier, 2004.

[6] M. Ghazel, M. Bigand and A. Toguyéni Exploitation des contraintes temporelles pour le suivi temps-réel des SEDs, journal Européen des Système Automatisés (JESA), vol. 39/2005, pp. 143-158, Ed. Hermes, 2005.

[7] M. Ghazel, A. Toguyéni and M. Bigand A semi-formal approach to build the functional graph of an automated production system for supervision purposes, International Journal of Computer Integrated Manufacturing (IJCIM), vol. 19, No 3, pp. 234-247, Ed. Taylor \& Francis, 2006.

[8] L. Khoudour, S. Ambellouis, C. Vieren, B. Fremont Détection d'obstacles devant les véhicules de transports guidés, Utilisation conjointe d'un radar coopératif et d'un stéréoscope optique, Revue I2M: Instrumentation, Mesure et Métrologie, février 2002, Edition Hermès. 